Article

\title{
A Real-Time Thermal Model for the Analysis of Tire/Road Interaction in Motorcycle Applications
}

\author{
Flavio Farroni ${ }^{1, * \mathbb{D}}$, Nicolò Mancinelli ${ }^{2}$ and Francesco Timpone ${ }^{1(\mathbb{C})}$ \\ 1 Dipartimento di Ingegneria Industriale-Università degli Studi di Napoli Federico II, 80138 Napoli NA, Italy; \\ francesco.timpone@unina.it \\ 2 Vehicle Dynamics, Ducati Motor Holding spa-Ducati Corse Division, 40132 Bologna BO, Italy; \\ nicolo.mancinelli@ducati.com \\ * Correspondence: flavio.farroni@unina.it; Tel.: +39-33-3374-2646; Fax: +39-08-1239-4165
}

Received: 4 February 2020; Accepted: 23 February 2020; Published: 28 February 2020

\begin{abstract}
While in the automotive field the relationship between road adherence and tire temperature is mainly investigated with the aim to enhance the vehicle performance in motorsport, the motorcycle sector is highly sensitive to such theme also from less extreme applications. The small extension of the footprint, along with the need to guarantee driver stability and safety in the widest possible range of riding conditions, requires that tires work as most as possible at a temperature able to let the viscoelastic compounds-constituting the tread and the composite materials of the whole carcass structure-provide the highest interaction force with road. Moreover, both for tire manufacturing companies and for single track vehicles designers and racing teams, a deep knowledge of the thermodynamic phenomena involved at the ground level is a key factor for the development of optimal solutions and setup. This paper proposes a physical model based on the application of the Fourier thermodynamic equations to a three-dimensional domain, accounting for all the sources of heating like friction power at the road interface and the cyclic generation of heat because of rolling and to asphalt indentation, and for the cooling effects because of the air forced convection, to road conduction and to turbulences in the inflation chamber. The complex heat exchanges in the system are fully described and modeled, with particular reference to the management of contact patch position, correlated to camber angle and requiring the adoption of an innovative multi-ribbed and multi-layered tire structure. The completely physical approach induces the need of a proper parameterization of the model, whose main stages are described, both from the experimental and identification points of view, with particular reference to non-destructive procedures for thermal parameters definition. One of the most peculiar and challenging features of the model is linked with its topological and analytical structure, allowing to run in real-time, usefully for the application in co-simulation vehicle dynamics platforms, for performance prediction and setup optimization applications.
\end{abstract}

Keywords: motorcycle tires; thermal modeling; performance optimization; real-time simulations

\section{Introduction}

A four-wheeled vehicle, even if often exerting a hyper static equilibrium, requires that its stability is guaranteed by an optimal adherence with road, allowing to satisfy safety, performance, and comfort requirements. If such evidence is fundamental for cars vehicle dynamics, the optimization of tire/road interaction becomes a key factor in motorcycles, and in particular considering racing ones, characterized by working with high roll angles and speed [1].

In a deeper analysis of tire/road interaction in motorsport sector, the focus moves necessarily to the optimization of the contact conditions in reference to the behavior of materials constituting tire tread and inner layers [2,3]. In particular, the adhesive bonding [4] and the power dissipated in the local 
indentation of road asperities [5], are highly influenced by the viscoelastic properties of tire polymers; such properties mainly vary with stress frequency [6], local displacement [7], and temperature [6,8].

The relationship between tire performance and temperature is a widely discussed topic [9-11], and racing tires, with the aim to exhibit an extra-ordinary frictional attitude, are designed adopting specific mixing of materials working at their best in a narrow thermal range. The challenge, for the driver and for the team engineers, is getting information on such thermal range to make sure that the tire spends the most of its time inside it, acting on proper vehicle setup, driving style and controls.

Moreover, several studies report that tire tread exhibits optimal grip depending on the temperature reached in its core layer $[12,13]$, not reachable by measurement instruments for thermal monitoring, and that global tire stiffness is highly influenced by the thermodynamic conditions of the carcass [14], also not directly measurable. For this reason, and for the increasing request of tools able to reproduce with high reliability the contact with road in a vehicle dynamics environment, the development of a tire thermal model has become a necessity for the players requiring the predictivity in challenging simulation scenarios.

In literature, the first approaches to such issue are related to the modeling of the Fourier equations applied to a three-dimensional domain, in some cases coupled with a mechanical model of the tire $[15,16]$, in other with stand-alone tools able to work together with other interaction formulations, like Pacejka's MF [17], or with FEM [18]. During the past years the focus has moved to highly discretized models able to work in real time [19], whose maximum level of complexity has been requested by motorcycle applications, for which tire contact patch moves along lateral direction of the tread, generating local stress [20] able to modify significantly the whole balance of energy with respect to car tires [19].

The paper describes the main structure and the approach followed in the thermodynamic modeling of a motorcycle racing tire, accounting for the parameterization of the diffusivity of the different layers, of the contact patch under variable working conditions, of the heat generation effects and of the conductive/convective interactions with the external environment, leading to a physical formulation for the real-time simulation of a virtual tire, conceived for a better knowledge of the mechanisms responsible for its forces exchange and for the development of performance optimization strategies linked to specific vehicle setup and boundary conditions management.

\section{2. thermoRIDE-Tire Thermodynamic Model}

thermoRIDE is a physical-analytical tire model, employable to study and understand all the phenomena concerning the tire during its interaction with both the external environment and the inner wheel chamber (inner air, rim, brakes, vehicle geometry, etc.)), as illustrated in Figure 1.
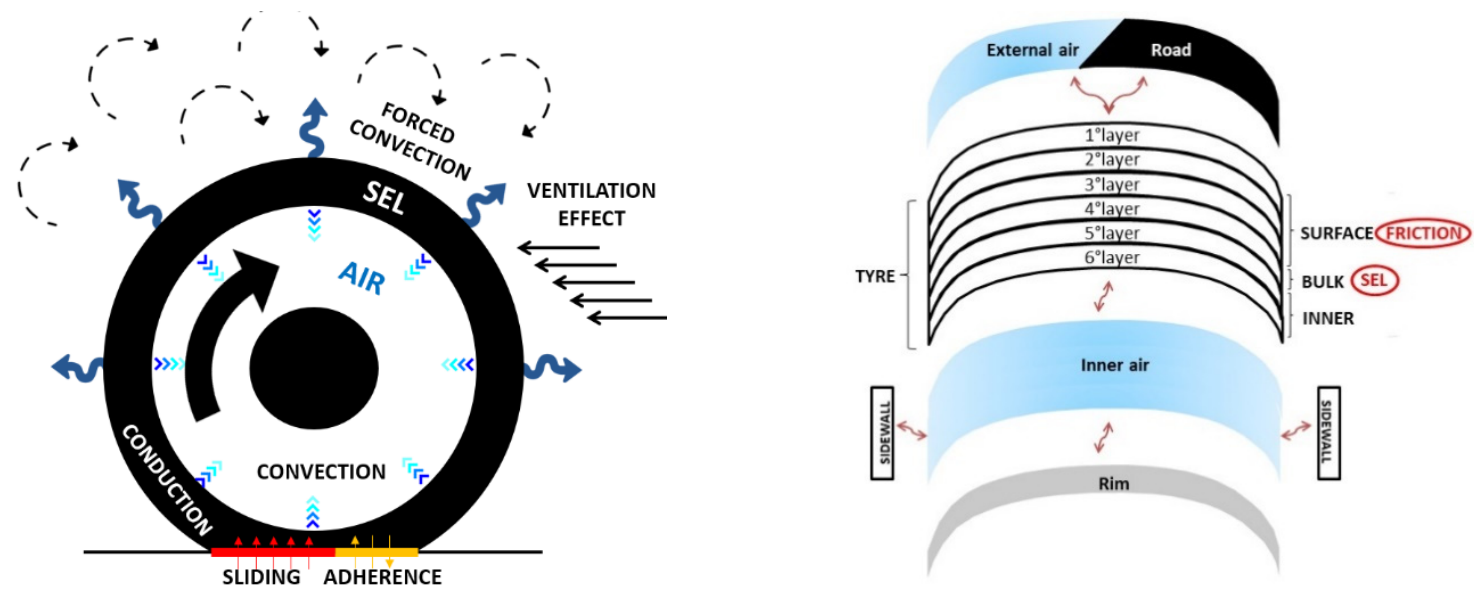

Figure 1. thermoRIDE model scheme. 
thermoRIDE model takes into account the following physical phenomena:

- Heat generation within the tire structure due to:

- $\quad$ Tire/road tangential interaction, known as FP (friction power);

- Effect of tire cyclic deformation during the tire rolling, known as SEL (strain energy loss).

- Heat exchange with the external environment due to:

- Thermal conduction between the tire tread and the road pavement;

- Thermal convection of the tread surface with the external air;

- Thermal convection of the inner liner surface with the inner air.

- Heat conduction between the tire nodes due to the temperature gradient.

\subsection{Mathematical Model}

thermoRIDE thermodynamic model is based on the use of the Fourier's diffusion equation applied to a three-dimensional domain.

It refers to energy contained within the system, excluding potential energy because of external forces fields and kinetic energy of motion of the system as a whole, and it keeps account of the gains and losses of energy of the system.

The law of heat conduction, also known as Fourier's law, states that the time rate of heat transfer through a material is proportional to the negative gradient in the temperature and to the area through which the heat flows. The differential form of Fourier's law shows that the local heat flux density $\vec{q}$ is equal to the product of thermal conductivity $\mathrm{k}$ and the negative local temperature gradient $\mathrm{VT}$. The heat flux density is the amount of energy that flows through a unit area per unit time:

$$
\vec{q}=-k * \nabla T
$$

where:

- $\quad q$ is the local heat flux density, in $\left[\mathrm{W} / \mathrm{m}^{2}\right]$;

- $\quad k$ is the material's conductivity, in $\left[\frac{W}{m * K}\right]$;

- $\quad \nabla T$ is the temperature gradient, in $\left[\frac{K}{m}\right]$.

It is possible to obtain a parabolic partial differential equation from the Fourier's law, especially useful for the numerical integration problems in transient thermal conditions. An infinitesimal volume element $d V=d x * d y * d z$ is considered in order to derive the system of the diffusion equations for each part of the tire structure.

Since the change in the internal energy of a closed system is equal to the amount of heat supplied to the system, minus the amount of work done by the system on its surroundings, and the control volume is considered not deformable, the internal energy $d U$ of the infinitesimal volume $d V$ is given by:

$$
d U=d m * c_{v} * T=\rho * d V * c_{v} * T
$$

where the volume cannot do any work $(d L=0)$, as assumed above. That is why the change in the internal energy $d U$ is considered only to the amount of heat $d Q$ added to the system.

The quantity $d Q$ stands for the heat supplied to the system by its surroundings and it takes into account two different contributions:

- Heat exchanged $d Q_{E X}=-d t * \oint_{d S} \vec{q} \cdot \vec{n} * d S$ through the outer surface of the volume $d V$;

- Heat generated $d Q_{G}=\dot{q}_{G} * d V * d t$ inside it. 
Therefore, the equation leads to:

$$
d Q=d Q_{E X}+d Q_{G}
$$

where:

- $\quad \dot{q}_{G}$ is the heat amount generated per unit time and per unit volume, in $\frac{W}{m^{3} * s}$;

- $\vec{n}$ is the normal unit vector respectively to the faces of the volume element.

For Gauss's divergence theorem, which postulates the equality between the flux of a vector field through a closed surface and the volume integral of the divergence over the region inside the surface, an integral taken over a volume $\oint_{d V} d i v \vec{q} * d V$ can replace the one taken over the surface bounding that volume $\oint_{d S} \vec{q} \cdot \vec{n} * d S$, as following:

$$
d Q_{E X}=-d t * \oint_{d S} \vec{q} \cdot \vec{n} * d S=-d t * \oint_{d V} d i v \vec{q} * d V
$$

In addition, the integral symbol can be avoided without affecting the physical meaning of the expression above, since the equation was carried out for an infinitesimal volume element $d V$ :

$$
d Q_{E X}=-d t * \operatorname{div} \vec{q} * d V
$$

Combining the Equations (4) and (5), it results:

$$
d Q_{E X}=d t * \operatorname{div}(k * \nabla T) * d V
$$

In conclusion, summing up (2), (3), and (5), the energy balance equation is obtained for the infinitesimal volume $d V$ :

$$
\rho * d V * c_{\mathcal{V}} * d T=\dot{q}_{G} * d V * d t+d t * \operatorname{div}(k * \nabla T) * d V
$$

which, divided both sides by the quantity $\rho * d V * c_{v} * d t$, defines the diffusion or heat equation of Fourier:

$$
\frac{\partial T}{\partial t}=\frac{\dot{q_{G}}}{\rho * c_{v}}+\frac{\operatorname{div}(k * \nabla T)}{\rho * c_{v}}
$$

Equation (8) allows to obtain the three-dimensional distribution of temperature $T(x, y, z, t)$, once the boundary condition are specified.

The Fourier's heat equation governs the temperature variation in time in relation to a special thermal gradient, and shows how the temperature will vary over time because of the generative effects or because of the ones linked to the heat transport.

In general, the Fourier's law allows studying only stationary thermal phenomena, whereas the heat diffusion equation also admits transient states. The complexity of the phenomena under study and the degree of accuracy required have made it necessary to take into account the dependence of the thermodynamic quantities and in particular of the thermal conductivity on the temperature. Furthermore, the non-homogeneity of the tire has made it necessary to consider the variation of the above parameters also along the thickness.

\subsection{Physical Model}

\subsubsection{Tire Structural Model}

Depending on the tire peculiar characteristics (dimensions, diffusivity, and inertia), the tire discretization can vary considerably with the main purpose to satisfy both the representation of all the 
physical phenomena characterizing transient and steady-state tire thermal dynamic and the necessity to preserve the hard real-time requirement in all the tire operating conditions.

The default tire discretization along the radial and lateral directions are respectively illustrated in Figure 2 on the left and right sides. However, the motorcycle tire discretization along the ISO $y$ direction can be freely modified up to 16 ribs ( 5 ribs default configuration is represented in the Figure 2) once the pre-initialized boundary conditions maps for the specific mesh configuration are available for the specific tire under analysis.
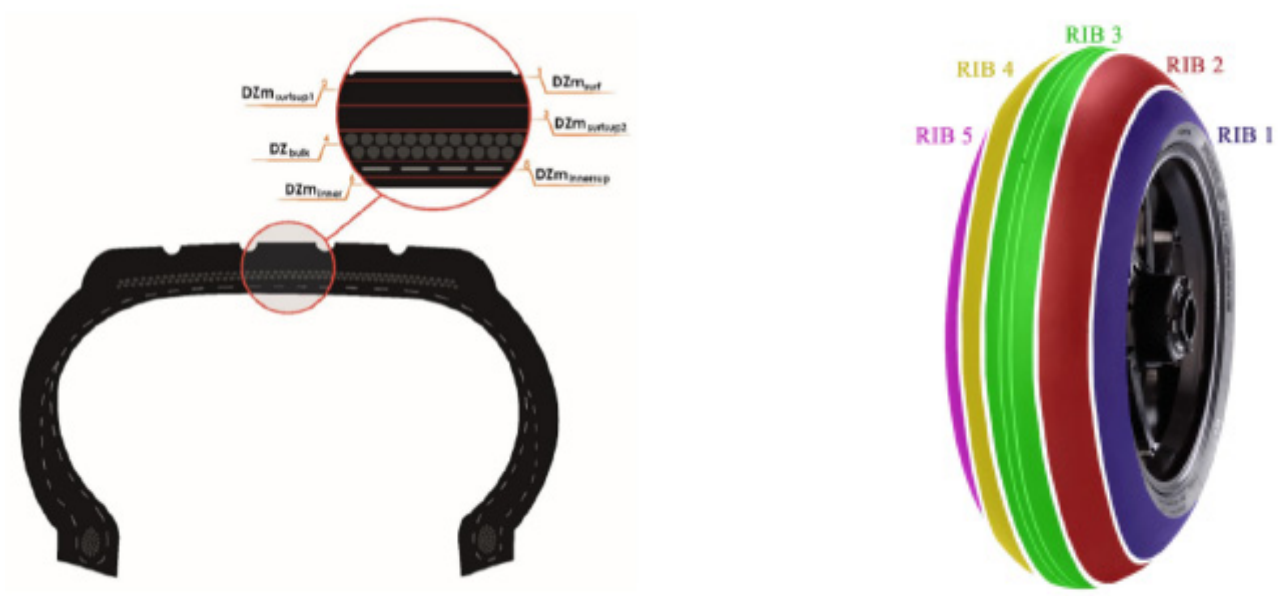

Figure 2. Example of thermoRIDE tire mesh configuration.

The default six layers of the tire structure along the radial direction are the followings:

- Tread surface, which is the most external part of the tread, the one which is in contact with the tarmac and the external air;

- Tread core is just below the surface and it is strictly connected with the grip level the tire is able to provide but it affects also the tire stiffness;

- Tread base is the deepest part of the tread, the last part before the belt; its temperature is more linked to the tire stiffness rather than the grip level;

- Belt is just below the Tread Base and it gives a big contribution to the SEL;

- Plies which is the last layer of the tire structure, it is another important contributor to the SEL, thanks to the energy dissipated by the friction among different plies and within the plies;

- Inner liner is the layer in contact with the inner air, which is not contributing to SEL dissipation neither linked to tire stiffness and grip.

\subsubsection{Contact Patch Evaluation}

The size and the shape of contact area are obtained by means of specifically developed test procedures, based on the pressure sensitive films or employing more complex models like multibody (MBD) or finished elements (FEA) ones.

Since the characterization procedures are linked to static application of vertical load $F_{z}$ in different conditions of internal pressure $P_{i}$ and wheel alignment configuration expressed in terms of camber $\gamma$, the contact patch configuration concerning its shape and extension refers to static load conditions in case of the employment of the sensitive film characterization methodology.

However, the instantaneous dynamic contact patch extension and shape can be rather different, because of particular transient conditions of wheel loading, centrifugal effect on the rolling tire, and viscoelastic tire intrinsic characteristics. The adoption of a MBD/FEA tire model able to fit both static and dynamic experimental data, respectively represented by vertical rim lowering and contact patch extension and shape in quasi-static conditions and strain energy loss cycle areas in different load and frequency conditions because of viscous properties of the tire structure, can constitute a valid 
instrument to implement the contact patch dynamic characteristics within the thermoRIDE model, by means of discretized areas associated to the control volume, as illustrated in Figure 3. Moreover, such models are capable to provide footprint areas depending on wheel travelling velocity or rolling frequency, affecting tire shape because of centrifugal effects.
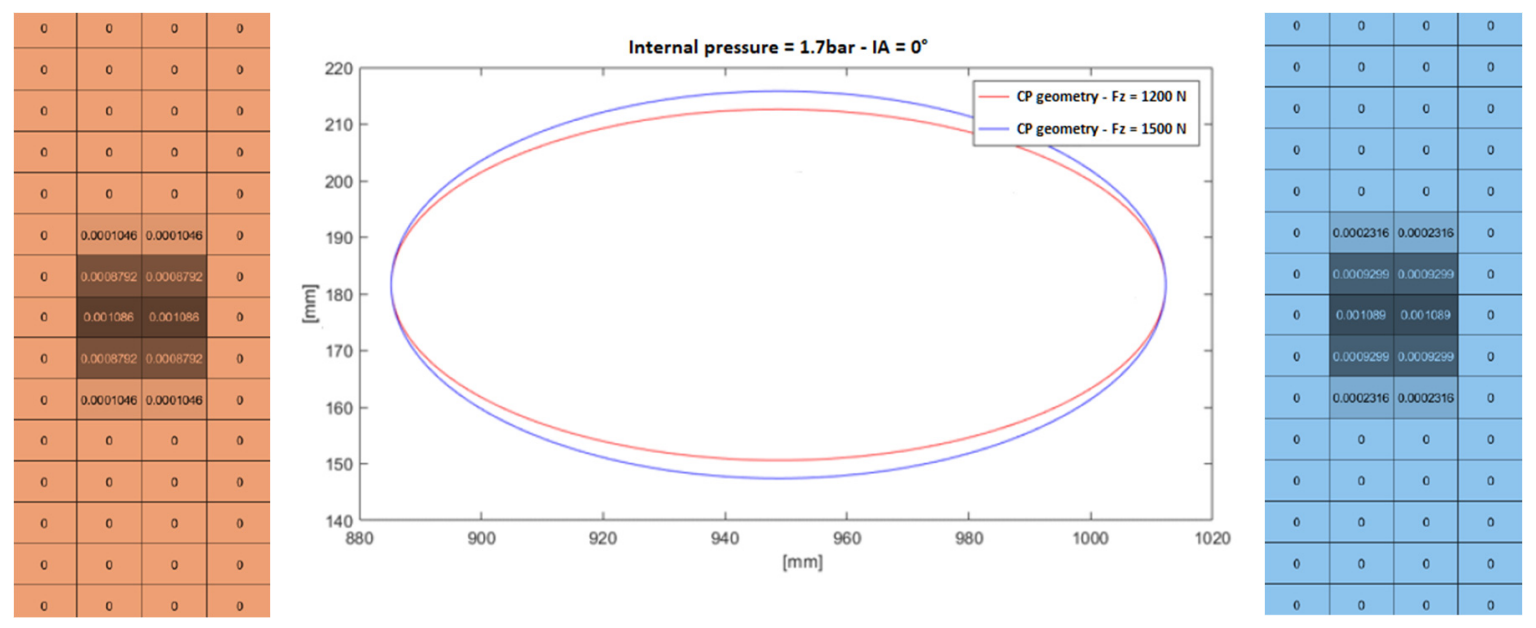

Figure 3. Example of thermoRIDE contact patch geometry representation.

At the current stage, thermoRIDE is able to adopt real contact patch areas from both the experimental activities (static, as said) or the MBD/FEM model outputs (static or dynamic, depending on the model), expressing thanks to parameters identification algorithms the footprint extension as a function of the main variables affecting its shape, as in the following:

$$
A_{C P}=f\left(F_{z}, P_{i}, \gamma\right)
$$

\subsubsection{Heat Exchange with Road Surface}

The thermal conductive exchange between the tread and the asphalt has been modeled through Newton's formula, schematizing the whole phenomenon by means of an appropriate coefficient of heat exchange. The term for such exchanges, for the generic i-th node will be equal to:

$$
Q_{C}=H_{c} \cdot\left(T_{r}-T_{i}\right) \cdot \Delta X \cdot \Delta Y
$$

where:

- $H_{c}$ is the convective heat transfer coefficient, estimated for the track testing conditions, in $\frac{W}{m^{2} \cdot K^{\prime}}$;

- $\quad T_{r}$ is the track temperature $[\mathrm{K}]$.

The heat generation at the tire-road interface is connected with the thermal power because of the tangential stresses that, in the sliding zone of the contact patch, dissipated in heat. Friction power can be associated directly to the nodes involved in the contact with the ground, and it is calculated as referred to global values of force and sliding velocity, assumed to be equal in the whole contact patch:

$$
F P=\frac{F_{x} \cdot v_{x}+F_{y} \cdot v_{y}}{A}
$$




\subsubsection{Heat Exchange with External/Inside Air}

The whole mechanism of the heat transfer between a generic surface and a moving fluid at different temperatures is described by natural and forced convection equations. The convection heat transfer is expressed by Newton's law of cooling, as before:

$$
h_{\text {conv }} \cdot\left(T_{\text {fluid }}-T_{i}\right) \cdot \Delta X \cdot \Delta Y
$$

Therefore, the heat exchange with the outside air is modeled by the mechanism of forced convection, occurring when there is relative motion between the motorcycle and the air, and by natural convection, when such motion is absent.

Natural convection is also employed to characterize the heat transfer of the inner liner with the inflating gas. The determination of the convection coefficient $h$, both forced $h_{\text {forc }}$ and natural $h_{\text {nat }}$, is based on the classical approach of the dimensionless analysis.

Supposing the tire invested by the air similarly to a cylinder invested transversely from an air flux, the forced convection coefficient is provided by the following formulation [8,9]:

$$
h_{\text {forc }}=\frac{K_{\text {air }}}{L} \cdot\left[0.0239 \cdot\left(\frac{V_{x} \cdot L}{v_{\text {air }}}\right)^{0.805}\right]
$$

in which:

- $K_{a i r}$ is air conductivity, evaluated at an average temperature between the effective air one and outer tire surface one, in $\left[\frac{\mathrm{W}}{\mathrm{m} \cdot \mathrm{K}}\right]$;

- $V_{x}$ is considered to be equal to the forward speed, in $\left[\frac{\mathrm{m}}{\mathrm{s}}\right]$;

- $v_{a i r}$ is the kinematic viscosity of air, in $\left[\frac{\mathrm{m}^{2}}{\mathrm{~s}}\right]$;

- $\quad L$ is the characteristic length of the heat transfer surface, in [m];

- $\quad T_{m, a i r}$ is the arithmetic mean between the temperatures of the tire outer surface and the external air in relative motion, in $[\mathrm{K}]$.

The values of $h_{\text {forc }}$ evaluated with the above approach are close to those obtained by means of CFD simulations for a motorcycle tire, represented in Figure 4.
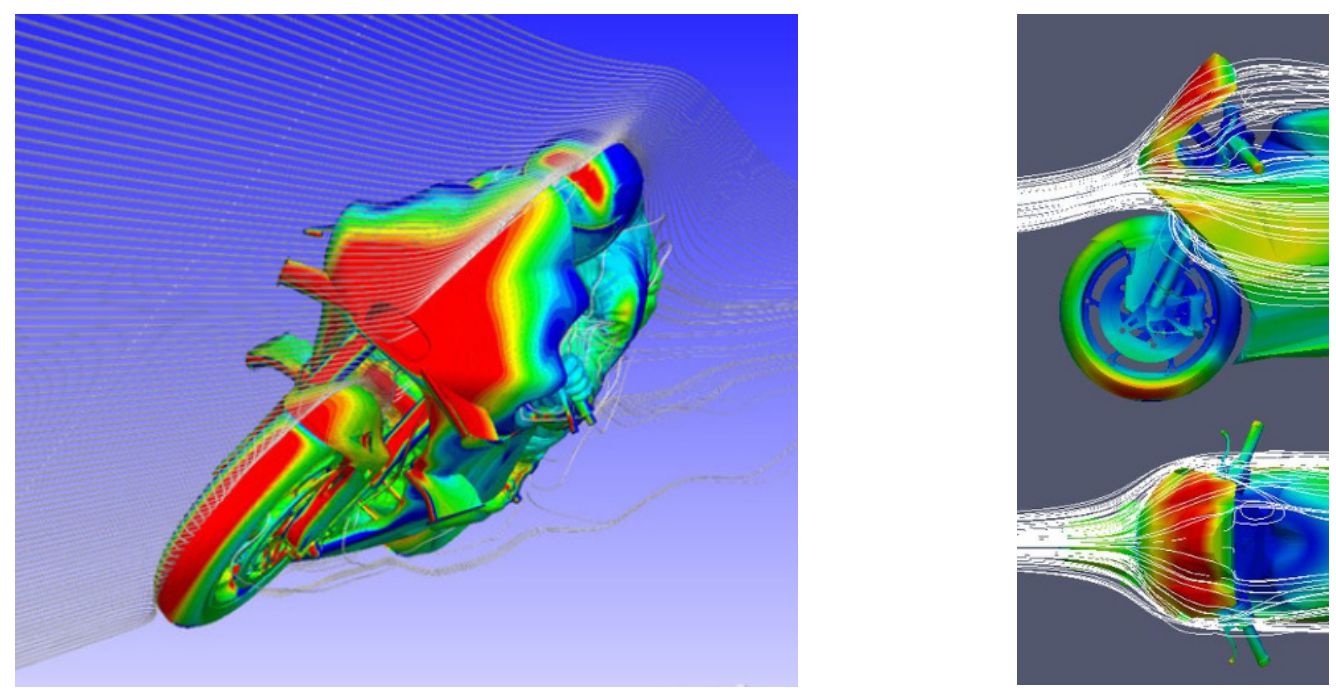

Figure 4. Example of motorcycle Computational Fluid Dynamics (CFD) simulations, useful for both front and rear tires convection parameterization. 
The natural convection coefficient $h_{\text {nat }}$, however, can be expressed as:

$$
h_{\text {nat }}=\frac{N u \cdot k_{a i r}}{L}
$$

in which, for this case:

$$
N u=0.53 \cdot G r^{0.25} \cdot \operatorname{Pr}^{0.25}
$$

\subsubsection{Hysteretic Generative Term}

The energy generated by the tire because of cyclic deformations is due to a superposition of several phenomena: intra-plies friction, friction inside singular plies, nonlinear viscoelastic behavior of all rubbery components, etc.

During the rolling, the entire tire is subjected to the cyclic deformations with a frequency corresponding to the tire rotational speed. During the motion, portions of tire, entering in sequence in the contact area, are subjected to deformations, which cause kinetic energy loss and heat dissipation.

The amount of heat generated by deformation (SEL) is estimated through experimental tests carried out deforming cyclically the tire in three directions (radial, longitudinal, and lateral).

Estimated energies do not exactly coincide with the ones dissipated in the actual operative conditions, as the deformation mechanism is different; it is however possible to identify a correlation between them on the basis of coefficients estimated from real data telemetry and from the specifically developed dynamic analysis involving MBD/FEA models.

At the current stage, the empirical SEL formulation is a function of the following parameters and it deeply depends on the tire characteristics:

$$
S E L=f\left(F, \omega, \gamma, P_{i}\right)
$$

whose further details and trends are available in [21] and where:

- $\quad F$ is the average interaction force at the contact patch, in [N];

- $\omega$ is the wheel rotation frequency, in $[\mathrm{rad} / \mathrm{s}]$;

- $\quad \gamma$ is the wheel alignment camber angle, in [rad];

- $P_{i}$ is the gauge pressure within the wheel internal chamber, in [bar].

\subsubsection{Model Input/Output Interface}

The input data required by the thermoRIDE model consists of the following telemetry channels, as summarized in Table 1.

Table 1. thermoRIDE model inputs.

\begin{tabular}{cc}
\hline Physical Quantity & Description \\
\hline $\mathrm{F}_{\mathrm{Z}}$ & Vertical interaction force \\
$\mathrm{F}_{\mathrm{x}}$ & Longitudinal interaction force \\
$\mathrm{F}_{\mathrm{y}}$ & Lateral interaction force \\
$\mathrm{v}_{\mathrm{x}}$ & Wheel hub longitudinal velocity \\
$\mathrm{s}_{\mathrm{r}}$ & Slip ratio \\
$\mathrm{s}_{\mathrm{a}}$ & Slip angle \\
$\omega$ & Wheel angular velocity \\
$\gamma$ & Inclination angle \\
$\mathrm{T}_{\text {air }}$ & Ambient air temperature \\
$\mathrm{T}_{\text {road }}$ & Road pavement temperature \\
\hline
\end{tabular}

Some of these data result from the telemetry measurements available for different tracks and are preliminarily analyzed in order to check their reliability; others, such as in particular the ones related 
to structural and thermal characteristics of the tire, are estimated on the basis of measurements and tests conducted on the tires.

In addition to tread and inner liner temperature distributions, as reported in Table 2, the model also provides the thermal flows involving the tire, such as the flow due to the external air cooling, the one due to the cooling with the road, the one with the inflation air, as well as the flows due to friction, hysteresis, and exchanges between the different layers.

Table 2. thermoRIDE model outputs.

\begin{tabular}{cc}
\hline Physical Quantity & Description \\
\hline $\mathrm{T}_{\text {treadSurf }}$ & Tread surface temperature \\
$\mathrm{T}_{\text {treadCore }}$ & Tread core temperature \\
$\mathrm{T}_{\text {treadBase }}$ & Tread base temperature \\
$\mathrm{T}_{\text {innerLiner }}$ & Inner liner temperature \\
$\mathrm{T}_{\text {innerAir }}$ & Internal air temperature \\
$\mathrm{P}_{\text {innerAir }}$ & Internal air pressure \\
$\mathrm{W}_{\text {extConvection }}$ & Ambient air convection \\
$\mathrm{W}_{\text {intConvection }}$ & Chamber air convection \\
$\mathrm{W}_{\text {extConduction }}$ & Road pavement conduction \\
$\mathrm{W}_{\text {longFriction }}$ & Longitudinal friction \\
$\mathrm{W}_{\text {latFriction }}$ & Lateral friction \\
$\mathrm{W}_{\text {sel }}$ & Strain Energy Loss \\
\hline
\end{tabular}

It has to be highlighted that, once per season, it is necessary to carry out an appropriate set of tuning factors to use it in a predictive manner.

The above procedure is linked with the vehicle specific configuration, connected with motorcycle setup and tires construction. Once the tuning phase is completed, known all vehicle data thermoRIDE inputs, the results obtained are in good agreement with the experimental data, with reference to the various operating conditions of the different tracks, allowing to adopt the model for analysis and supporting vehicle design determination.

\section{Results}

In the Figures 5 and 6 , the temperature trends of all the tire layers of the thermodynamic model are illustrated (it must be highlighted that in the above figures the temperature values are dimensionless because of confidentiality agreements with industrial partners). It has to be clarified that the measured data in Figure 5 in red (acquired from external IR sensors) report the temperature of fixed points along the lateral direction of the tire tread, while the simulated ones, in blue, are related to each one of the 15 ribs available, in any moment of the run. 


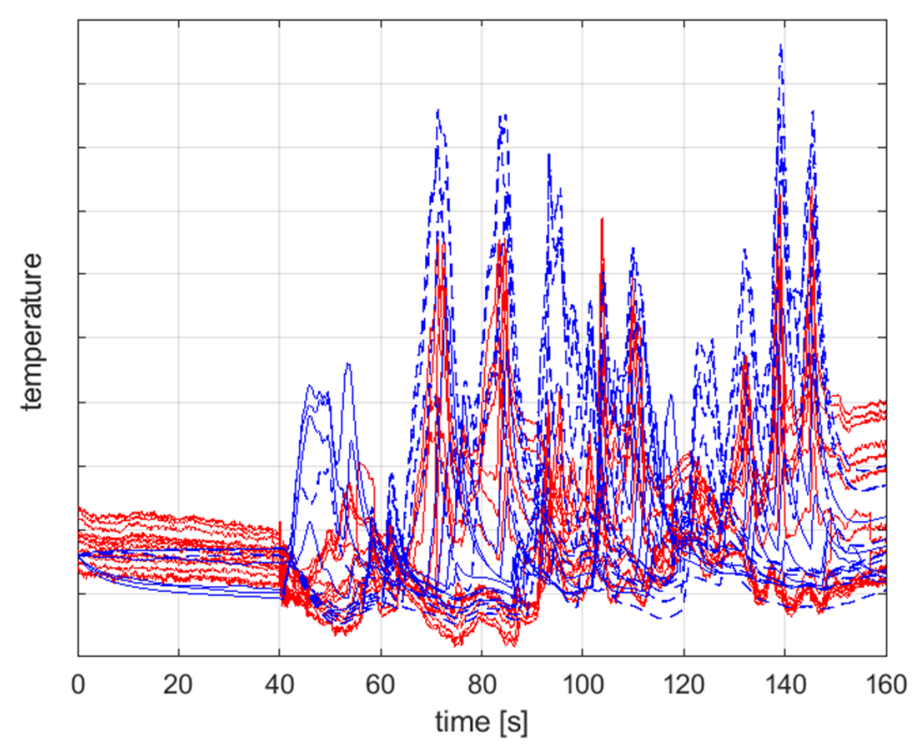

Figure 5. Comparison between the tread external temperatures obtained by means of the 16-ribs' configuration model (in blue) and the acquired ones (in red).

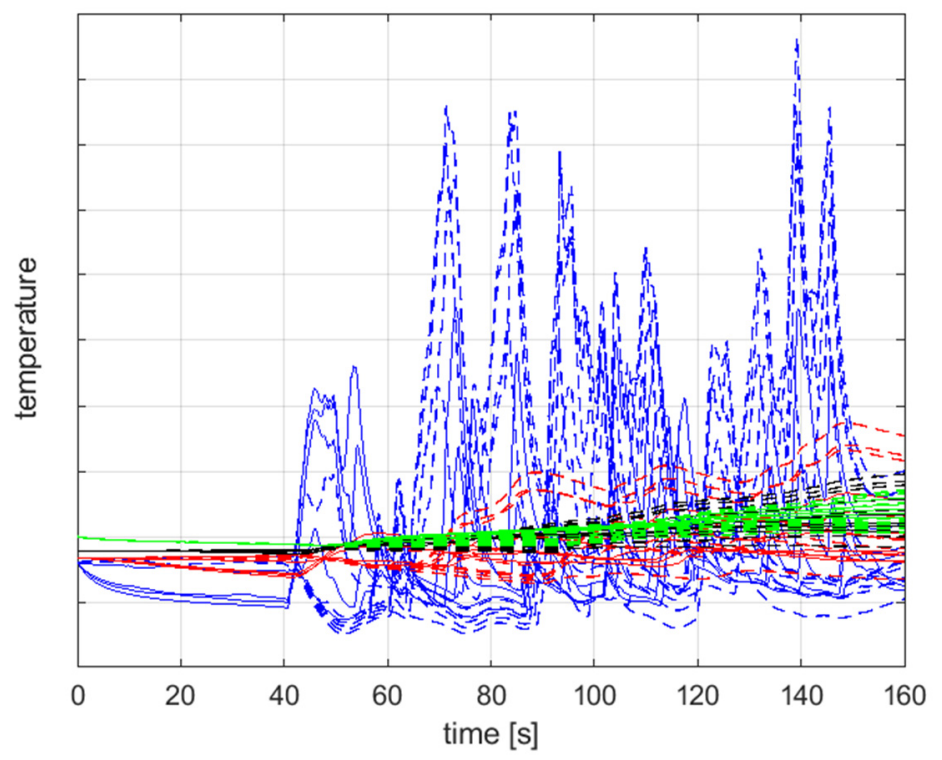

Figure 6. Tire temperatures of all the tire layers obtained by means of the thermoRIDE model: tread surface (in blue), tread core (in red), tread base (in black) and innerliner (in green).

The difference in the thermal shapes of the external layers is due to their position inside the tire structure: the tread layers, especially the surface one, are subjected to the instant thermal powers generated by the tire/road interaction and convective flows; meanwhile a slow temperature trend induced concurrently by the rolling fatigue effect and by the convective heat exchanges characterizes the internal layers' dynamics. That is why, the internal tire layers seem to have a slow temperature ascent during the rolling motion of the wheel, while the tread surface is characterized by an oscillating profile.

The ability to predict the interior temperature distribution, and thus the grip behavior of the tire, is fundamental in terms of the vehicle handling improvement and of the asset optimization according to highly variable outdoor testing conditions.

Vehicle optimal setup is deeply linked with the correct compound working conditions, as highlighted in Figure 7 for a reference automotive tire, not directly linked to the described motorcycle thermal model, but able to show expected physical trends. Such optimization is achievable with a 
proper suspension layout in terms of stiffness, compliance, and of suitable wheel alignment geometry. In particular, owing to the availability of information on the time by time tread core temperature, plotted on the $x$-axis, it becomes clear that the tire shows an optimal grip in a specific thermal range that results to be the variable for each different compound, useful to be known in order to set an optimal configuration of the vehicle in any condition.

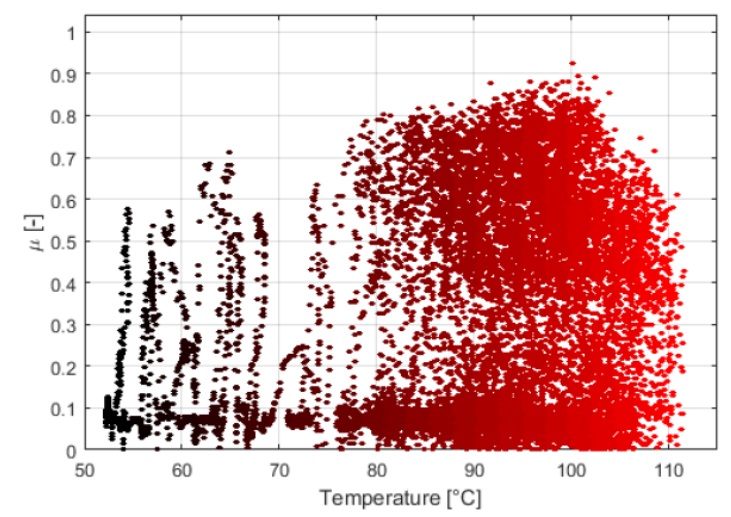

Figure 7. Tread core compound grip-temperature dependence.

Differences in terms of interaction characteristics (Figure 8), in which a considerable stiffness decrease because of a higher tire temperature is clearly appreciable, are shown both for longitudinal and lateral tire interaction curves.
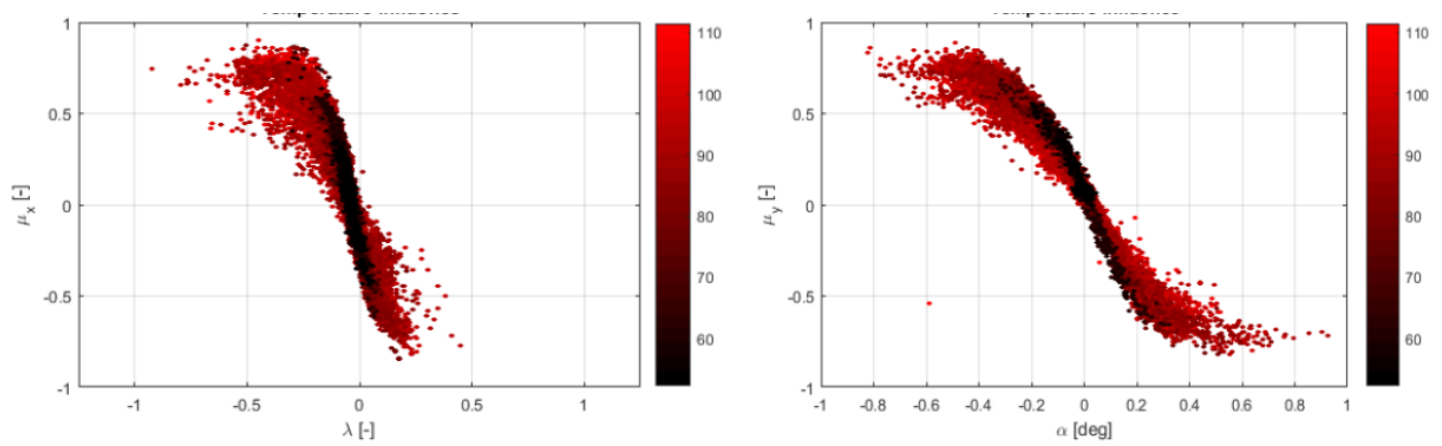

Figure 8. Temperature influence on tire interaction characteristics curves (longitudinal interaction on the left and lateral interaction on the right).

The influence of a large amount of parameters can be evaluated, such as, inclination angle, inflation pressure, different track and weather conditions, and the influence of the manufacturer's vehicle settings. To exploit the entire amount of grip available on the tire-road interface in order to preserve the highest level of handling performance preventing the tire from sliding, more and more physical phenomena regarding the vehicle and its subcomponents have to be taken into account within the integrated vehicle control systems.

\section{Conclusions}

The paper focuses on the development of a specific version of a tire thermal model for motorcycle applications. The main differences from the common thermodynamic models are related to the particular management of contact patch, responsible for the fundamental friction generation phenomena and for conduction with road, and that in motorcycle tires is characterized by particular elliptical and arched shape, in continuous motion along the lateral direction because of high roll/camber angle. 
Such peculiar behavior required a dedicated modeling approach, coupled with the possibility to implement micro-hysteresis differences in each single rib, referred to the possibility to adopt different compounds in the same tire, very common in motorsport.

The results of simulations carried out by coupling the tire thermal model with a vehicle one have been reported, showing a significant good agreement with the experimental results coming from dedicated outdoor acquisitions on an instrumented testing motorcycle. Once validated the model calibration, the main advantages of a predictive and reliable thermal model are the evaluation of the thermal fluxes and temperature interesting the inner layers of the tire structure (deeply linked to grip and stiffness variations, highly influencing vehicle performances and ride/comfort dynamics) and the possibility to study the vehicle setup and aerodynamics, owing to the estimation of the exact amount of energy to be generated/subtracted to let the tire work in the optimal thermal range.

Further development stages are concerning the implementation of the tread wear mechanisms, correlated with the energy provided to the tire lap by lap and deeply influencing the temperature distribution during the race event. Moreover, the real-time availability of knowledge on local temperature, coupled with reliable interaction forces estimation, is a key factor to the realization of MiL (model in the loop), HiL (hardware in the loop), and DiL (driver in the loop) simulation scenarios.

Author Contributions: For research articles with several authors, a short paragraph specifying their individual contributions must be provided. The following statements should be used "conceptualization, F.F., N.M. and F.T.; software, F.F., N.M.; validation, N.M.; data curation, N.M..; writing-original draft preparation, F.F.; writing-review and editing, F.T. All authors have read and agreed to the published version of the manuscript.

Funding: This research received no external funding.

Conflicts of Interest: The authors declare that they have no conflict of interest.

\section{References}

1. Cossalter, V.; Lot, R.; Massaro, M. Modelling, Simulation and Control of Two-Wheeled Vehicles; John Wiley \& Sons: Hoboken, NJ, USA, 2014; pp. 1-42.

2. Gargallo, L.; Radic, D. Physicochemical Behavior and Supramolecular Organization of Polymers; Springer Science \& Business Media: Berlin/Heidelberg, Germany, 2009; pp. 43-162.

3. Smith, R.H. Analyzing Friction in the Design of Rubber Products and Their Paired Surfaces; CRC Press: Boca Raton, FL, USA, 2008.

4. Heinrich, G.; Klüppel, M. Rubber Friction, Tread Deformation and Tire Traction. Wear 2008, 265, 1052-1060. [CrossRef]

5. Farroni, F.; Russo, R.; Timpone, F. Theoretical and Experimental Estimation of the Hysteretic Component of Friction for a Visco-Elastic Material Sliding on a Rigid Rough Surface. Int. Rev. Mech. Eng. 2013, 6, 3.

6. Ferry, J.D.; Myers, H.S. Viscoelastic Properties of Polymers. J. Electrochem. Soc. 2007, 41, 53-62.

7. Radford, D.W.; Fitzhorn, P.A.; Senan, A.; Peterson, M.L. Application of Dynamic Mechanical Analysis to the Evaluation of Tire Compounds. SAE Technical Pap. Ser. 2002, 111, 2492-2496.

8. Kern, W.J.; Futamura, S. Effect of Tread Polymer Structure on Tyre Performance. Polymer 1988, 29, 1801-1806. [CrossRef]

9. Mavros, G. A Thermo-Frictional Tyre Model Including the Effect of Flash Temperature. Veh. Syst. Dyn. 2019, 57, 721-751. [CrossRef]

10. Segers, J. Analysis Techniques for Racecar Data Acquisition, 2nd ed.; SAE Technical Paper: Warrendale, PA, USA, 2014.

11. Sharp, R.S.; Gruber, P.; Fina, E. Circuit Racing, Track Texture, Temperature and Rubber Friction. Veh. Syst. Dyn. 2016, 54, 510-525. [CrossRef]

12. Angrick, C.; van Putten, S.; Prokop, G. Influence of Tire Core and Surface Temperature on Lateral Tire Characteristics. SAE Int. J. Passeng. Cars Mech. Syst. 2014, 7, 468-481. [CrossRef]

13. Farroni, F.; Sakhnevych, A.; Timpone, F. Physical Modelling of Tire Wear for the Analysis of the Influence of Thermal and Frictional Effects on Vehicle Performance. J. Mater. Des. Appl. 2017, 231, 151-161. [CrossRef]

14. Kelly, D.P.; Sharp, R.S. Time-Optimal Control of the Race Car: Influence of a Thermodynamic Tyre Model. Veh. Syst. Dyn. 2012, 50, 641-662. [CrossRef] 
15. Ozerem, O.; Morrey, D. A Brush-Based Thermo-Physical Tyre Model and Its Effectiveness in Handling Simulation of a Formula SAE Vehicle. J. Automob. Eng. 2019, 233, 107-120. [CrossRef]

16. Février, P.; Blanco Hague, O.; Schick, B.; Miquet, C. Advantages of a Thermomechanical Tire Model for Vehicle Dynamics. ATZ Worldw. 2010, 112, 33-37. [CrossRef]

17. Farroni, F.; Giordano, D.; Russo, M.; Timpone, F. TRT: Thermo Racing Tyre a Physical Model to Predict the Tyre Temperature Distribution. Meccanica 2014, 49, 707-723. [CrossRef]

18. Wang, Z. Finite Element Analysis of Mechanical and Temperature Field for a Rolling Tire. In Proceedings of the IEEE 2010 International Conference on Measuring Technology and Mechatronics Automation, Changsha, China, 13-14 March 2010; IEEE: Piscataway, NJ, USA.

19. Farroni, F.; Russo, M.; Sakhnevych, A.; Timpone, F. TRT EVO: Advances in Real-Time Thermodynamic Tire Modeling for Vehicle Dynamics Simulations. J. Automob. Eng. 2019, 233, 121-135. [CrossRef]

20. Cossalter, V.; Doria, A. The Relation between Contact Patch Geometry and the Mechanical Properties of Motorcycle Tyres. Veh. Syst. Dyn. 2005, 43, 156-164. [CrossRef]

21. Farroni, F.; Rocca, E.; Timpone, F. A Full Scale Test Rig to Characterize Pneumatic Tyre Mechanical Behaviour. Int. Rev. Mech. Eng. 2013, 7, 841-846.

(C) 2020 by the authors. Licensee MDPI, Basel, Switzerland. This article is an open access article distributed under the terms and conditions of the Creative Commons Attribution (CC BY) license (http://creativecommons.org/licenses/by/4.0/). 